\title{
More than insulator: multiple roles of CTCF at the H19-Igf2 imprinted domain
}

\author{
Purnima Singh, Dong-Hoon Lee ${ }^{\dagger}$ and Piroska E. Szabó* * \\ Department of Molecular and Cellular Biology, Beckman Research Institute, Duarte, CA, USA
}

Edited by:

Michèle Amouyal, Centre National de la Recherche Scientifique, France

Reviewed by:

Michèle Amouyal, Centre National de la Recherche Scientifique, France Patrick McGowan, Duke

University, USA

\section{*Correspondence:}

Piroska E. Szabó, Department of Molecular and Cellular Biology, Beckman Research Institute, City of Hope, 1500 E Duarte Rd, Duarte, CA 91010, USA.

e-mail:pszabo@coh.org

${ }^{+}$Present address:

Dong-Hoon Lee, Department of Genetics and Biochemistry,

Clemson University, Clemson, USA.
CTCF (CCCTC-binding factor)-mediated insulation at the H19-Insulin-like growth factor 2 (lgf2) imprinted domain is a classic example for imprinted gene regulation. DNA methylation difference in the imprinting control region (ICR) is inherited from the gametes and subsequently determines parental allele-specific enhancer blocking and imprinted expression in the soma. Recent genetic studies showed that proper monoallelic enhancer blocking at the H19-lgf2 ICR is critical for development. Strict biallelic insulation at this locus causes perinatal lethality, whereas leaky biallelic insulation results in smaller size but no lethality. Apart from enhancer blocking, CTCF is also the master organizer of chromatin composition in the maternal allele along this imprinted domain, affecting not only histone tail covalent modifications but also those in the histone core. Additionally, CTCF binding in the soma protects the maternal allele from de novo DNA methylation. CTCF binding is not involved in the establishment of the gametic marks at the ICR, but it slightly delays de novo methylation in the maternally inherited ICR allele in prospermatogonia. This review focuses on the developmental and epigenetic consequences of CTCF binding at the H19-lgf2 ICR.

Keywords: CTCF chromatin, imprinting, H19, Igf2, insulators, methylation, Zfp57, Trim28
CTCF (also known as CCCTC-binding factor) is a major organizer of the vertebrate genome and is essential for development (Moore et al., 2012). It is a versatile protein that regulates gene expression by binding to DNA via its multiple zinc fingers (Filippova, 2008; Ohlsson et al., 2010; Herold et al., 2012). CTCF plays roles in transcriptional activation and repression, insulation by enhancer blocking or chromosome barrier formation and organization of higher order chromatin by chromosomal looping and nuclear tethering (Phillips and Corces, 2009; Weth and Renkawitz, 2011; Barkess and West, 2012; Ghirlando et al., 2012). CTCF has been implicated in such diverse biological phenomena as genomic imprinting, $\mathrm{X}$ chromosome inactivation (Spencer et al., 2011), alternative splicing (Shukla et al., 2011), microsatellite instability (Libby et al., 2008), and V(D)J recombination (Guo et al., 2011). Several methodologies have been utilized for testing CTCF's function, including in vitro and cell culture assays, depletion or ablation of CTCF and its interactive partners, and deleting CTCF sites from episomal vectors, integrated transgenes or endogenous loci. The most direct functional test is to specifically inactivate the CTCF binding site(s) at an endogenous locus by point mutations. To date almost no such genetic studies exist in the latter category. One notable exception is the mouse H19Igf2 imprinted domain, which has been extensively studied in the past decade by several independent groups including ours. Precise point mutations have been made that inactivated the CTCF binding sites in the imprinting control region (ICR). In this review we will focus on some of the colorful roles that CTCF plays at the H19-Igf2 imprinted locus. We will review that CTCFmediated insulation controls reciprocal parental allele-specific expression of these two imprinted genes, emphasizing that correct monoallelic enhancer blocking at this locus is critical for normal fetal development. We will also summarize the roles CTCF plays in maintaining the epigenetic features of the maternal allele in the soma and, to some extent, in primordial germ cells (PGCs).

\section{PARENTAL ALLELE-SPECIFIC ENHANCER INSULATION AT THE H19-Igf2 IMPRINTED DOMAIN}

CTCF-mediated insulation is a classic example for the regulation of genomic imprinting. Imprinted genes exhibit parental allele-specific expression (Ferguson-Smith, 2011; Abramowitz and Bartolomei, 2012). Insulin-like growth factor 2 (Igf2), and H19 are neighboring genes, located on distal chromosome 7 in the mouse and expressed from the paternally or maternally inherited chromosome, respectively. Igf2 protein is important for promoting fetal and placental growth (DeChiara et al., 1990; Constancia et al., 2002) whereas the H19 non-coding RNA moderates growth in the normal fetus (Gabory et al., 2009), puts the brake on the growth of the term placenta via its microRNA (Keniry et al., 2012) and also functions as a tumor suppressor (Yoshimizu et al., 2008). Both genes respond to the same endodermal enhancers that are distal to H19 (Leighton et al., 1995) (Figure 1A). Between these two genes lies a $2.4 \mathrm{~kb}$ long differentially methylated region (DMR) that is required for the monoallelic expression of both the H19 and Igf2 genes, and therefore is called an ICR. Its deletion from the maternal allele results in biallelic Igf2 expression and from the paternal allele in biallelic H19 expression. Methylation of this DMR is exclusive to the paternally inherited chromosome and originates from the sperm (Tremblay et al., 1995, 


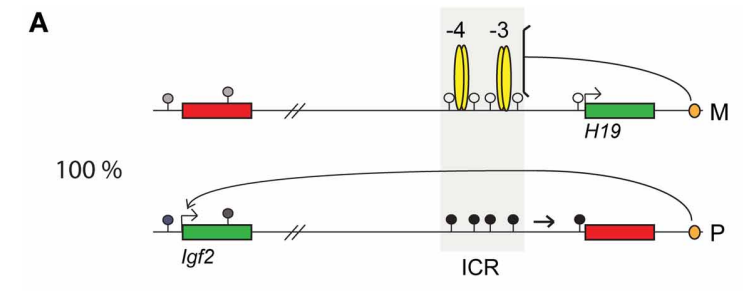

B

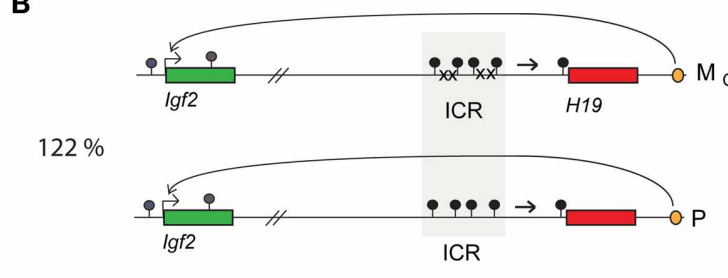

C

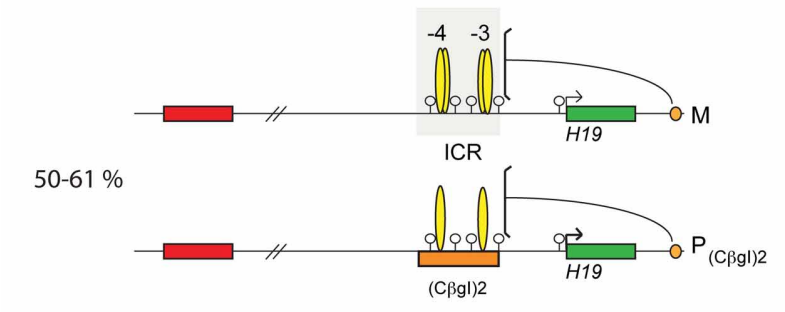

D

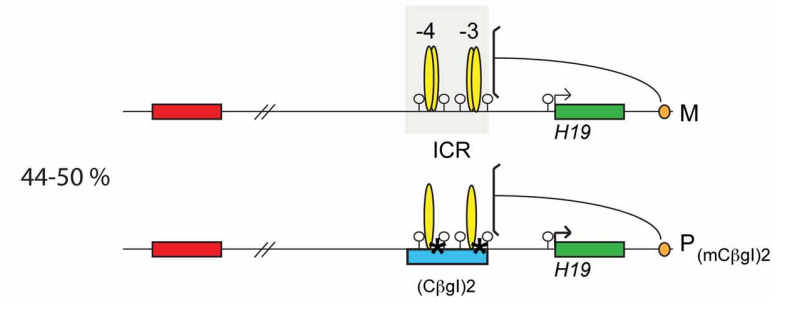

FIGURE 1 | Parental allele-specific enhancer insulation at the H19-Igf2 imprinted domain. (A) Imprinted insulation at the H19/lgf2 imprinted domain by CTCF binding in the ICR based on publications referenced in the text. Maternal chromosome (M): unmethylated (white lollipops) ICR (shaded area) is inherited from the oocyte. CTCF (yellow ovals) imparts insulator activity (bracket) between the lgf2 promoters and the shared, downstream enhancers (orange oval). Initiation of H19 expression depends on an unmethylated ICR during embryogenesis. Paternal chromosome (P): methylated (black lollipops) ICR is inherited from the sperm, CTCF cannot bind, hence ICR has no insulator activity thus, the Igf2 promoters and enhancers can interact. During early postimplantation development, the H19 promoter is inactivated by an ICR-dependent mechanism (horizontal arrow). (B) CTCF binding site mutations in the maternal ICR allele disrupt imprinted expression (Szabó et al., 2004). CTCF no longer binds in the mutant maternal chromosome ( $\mathrm{M}_{\mathrm{CTCFm}}$ ), thus, the enhancers can access the Igf2 promoter in both alleles. The mutant ICR is methylated and inactivates the $H 19$ promoter. (C) Non-imprinted insulation at the H19/lgf2 locus by the chicken $\beta$-globin insulator duplex $(\mathrm{Ch} \beta \mathrm{GI})_{2}$ (orange rectangle) (Szabó et al., 2002). The $(\mathrm{Ch} \beta \mathrm{Gl})_{2}$ is unmethylated and insulates the Igf2 promoter from the shared enhancers when substituted for the ICR and transmitted maternally (not shown) or paternally (P), with $10 \%$ Igf2 activity remaining. $\mathrm{H} 19$ is overactivated 1.5 -fold by the $(\mathrm{Ch} \beta \mathrm{Gl})_{2}$ sequences in the paternal allele (bold arrow). (D) Biallelic insulation by the mutant chicken

(Continued)

\section{FIGURE 1 | Continued}

$\beta$-globin insulator duplex $(\mathrm{mCh} \beta \mathrm{Gl})_{2}$ (turquoise rectangle) carrying mutations for boundary factor binding sites (stars) (Lee et al., 2010). Insulation is complete, with no detectable remaining Igf2 expression. Relative fetus size for each genotype is shown to the left. Active genes and silent genes are depicted with green and red rectangles, respectively.

1997; Thorvaldsen et al., 1998). Igf2 expression is also regulated by two additional paternally methylated DMRs. Igf2 DMR1, upstream of the Igf2 gene functions as a mesodermal silencer in the maternal allele (Constancia et al., 2000) while DMR2, in the sixth exon, functions as an enhancer in the paternal allele (Murrell et al., 2001).

To shed light on how the ICR regulates reciprocal expression of $I g 2$ and H19, we used in vivo DNAseI, DMS footprinting and UV photofootprinting analysis of mouse embryo fibroblasts (MEFs) carrying maternal or paternal duplication of distal Chromosome 7 and discovered strong footprints at four consensus CTCF binding sites in the unmethylated maternal ICR allele but not in the methylated paternal allele. This provided evidence that the CTCF insulator protein blocks communication between the Igf2 promoters and the shared downstream enhancers in the maternal chromosome (Szabó et al., 2000). At the same time, in vitro enhancer blocking, gelshift, episome assays, and in vivo ChIP assays confirmed that the H19-Igf2 ICR acts as an enhancer blocker in the unmethylated maternal allele and CTCF binding is inhibited in the paternal ICR allele by DNA methylation, allowing Igf2 promoter access to the enhancers (Bell and Felsenfeld, 2000; Hark et al., 2000; Kanduri et al., 2000). To verify the enhancer blocker role of CTCF at this locus in vivo, CTCF-site mutations were introduced into the ICR allele in the mouse. Maternal transmission of these mutations resulted in biallelic Igf2 expression and biallelic H19 silencing (Figure 1B) (Pant et al., 2003; Schoenherr et al., 2003; Szabó et al., 2004; Han et al., 2008). CTCF has also been reported to be responsible at this locus for asynchronous replication of the two alleles: late replication of the maternal allele depends on CTCF binding (Bergstrom et al., 2007; Guibert et al., 2012). CTCF-dependent enhancer blocking requires cohesins (Rubio et al., 2008; Stedman et al., 2008; Nativio et al., 2009; Yao et al., 2010; Xiao et al., 2011) and involves regulating chromosome loop formation (Murrell, 2011).

Parental allele-specific CTCF binding has been detected recently at additional imprinted domains, at the Rasgrf1 (Yoon et al., 2005), Gtl2 (Lin et al., 2011), Grb10 (Hikichi et al., 2003), Kcnq1/Kcnq1ot1 (Fitzpatrick et al., 2007), and Peg13 DMRs (Singh et al., 2011). It will be very interesting to test using genetic analyses whether these CTCF binding sites are required for regulating the allele-specific expression of imprinted transcripts by enhancer blocking.

\section{MONOALLELIC INSULATION AT THE H19-Igf2 ICR IS ESSENTIAL FOR NORMAL DEVELOPMENT}

Genetic studies revealed that insulation strength of the H19-Igf2 ICR has consequences to body size and viability. Insulation was absent at the H19-Igf2 domain in mice carrying the ICR CTCF 
site mutations in the maternal chromosome. This resulted in elevated Igf2 expression and an overgrowth phenotype (Figure 1B). Prenatal fetuses were $122 \%$ heavier than their normal siblings (Szabó et al., 2004). We also noticed that adult males that carried the ICR CTCF site mutations became aggressive and fought frequently. Insulation was biallelic at this locus in mice where the ICR was replaced with two copies of the chicken beta globin insulator $(\mathrm{Ch} \beta \mathrm{GI})_{2}$ (Figure 1C) (Szabó et al., 2002). This introduced DNA fragment was of similar size to the ICR, had two CTCF binding sites, and also included sufficient number of $\mathrm{CpG}$ dinucleotides. The $(\mathrm{Ch} \beta \mathrm{GI})_{2}$ functioned as an enhancer blocker in the maternal allele. In the paternal allele, however, it behaved differently from the endogenous ICR. The $(\mathrm{Ch} \beta \mathrm{GI})_{2}$ did not attain de novo methylation in the male germ line and thus, it was not methylated in the paternally inherited allele in the somatic organs of $+/(\mathrm{Ch} \beta \mathrm{GI})_{2}$ fetuses. It consequently allowed biallelic CTCF binding and insulation of the Igf2 promoters from the shared enhancers. Igf2 expression was reduced to $10 \%$ of normal values and fetus size was reduced to $50-61 \%$ of normal littermates. H19 expression was biallelic. Later a very similar mouse model was generated (Lee et al., 2010) that carried a mutant form of the (mCh $\beta \mathrm{GI})_{2}$ sequences (Figure 1D). CTCF binding sites were retained in the $(\mathrm{mCh} \beta \mathrm{GI})_{2}$ but consensus sites for boundary proteins, USF1 (West et al., 2004; Yao et al., 2010) and VEZF1 (Clark et al., 1990; Dickson et al., 2010), were destroyed by point mutations. Although there was a slight, 32\%, methylation at these sequences in the male germ line, paternal allele-specific methylation was not maintained in the soma. In $+/(\mathrm{mCh} \beta \mathrm{GI})_{2}$ offspring insulation was again biallelic, and even more strict than the insulation in $+/(\mathrm{Ch} \beta \mathrm{GI})_{2}$ fetuses. Igf2 expression was undetectable and fetus size was reduced to $44-50 \%$ of normal littermates. Whereas the $+/(\mathrm{Ch} \beta \mathrm{GI})_{2}$ mice were viable, a fully penetrant perinatal lethality occurred in the $+/(\mathrm{mCh} \beta \mathrm{GI})_{2}$ genotype (Figure 2A). The absence of Igf2 likely contributed to the lethality phenotype of $+/(\mathrm{mCh} \beta \mathrm{GI})_{2}$, but was not the sole cause, because Igf2 homozygous mutant mice are small but viable (DeChiara et al., 1990). Similar conclusion was reached in the reciprocal experiment (Figure 2B), when perinatal lethality of mice carrying maternal duplication of distal chromosome 7 (MatDup.dist7) was rescued by introducing the CTCF site mutations into one allele of the H19-Igf2 ICR (also called IC1) (Han et al., 2010). Correcting biallelic insulation of the H19-Igf2 ICR was sufficient to rescue lethality, even though the duplicated chromosome region of MatDup.dist7 mice also carries the Kcnq1ot1 maternally methylated DMR (also called IC2), and additional misexpressed imprinted genes. These results have revealed that correct insulator dose and strength at the H19-Igf2 ICR is required for perinatal viability: strict biallelic insulation at this imprinted locus is not tolerated in development.

\section{CTCF IS THE MAJOR EPIGENETIC ORGANIZER OF THE MATERNAL ALLELE IN THE SOMA}

CTCF is the master organizer of the maternal allele's chromatin (Figure 3). Utilizing single nucleotide polymorphisms (SNPs) between parental mouse lines and using quantitative allele-specific chromatin immunoprecipitation single nucleotide primer extension (SNuPE) assays, we measured the chromatin

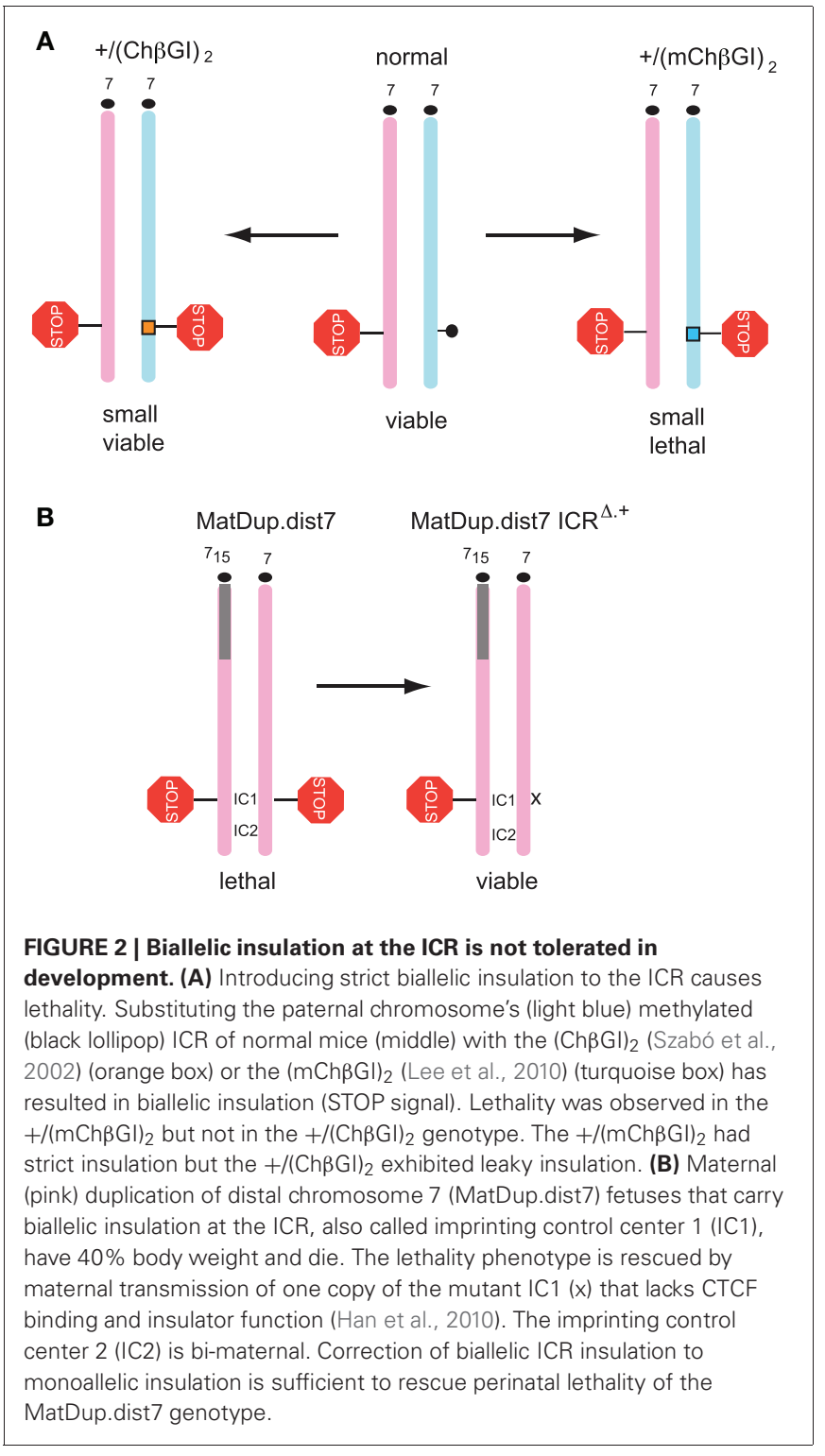

composition along the H19/Igf2 imprinted domain in normal cells and cells with engineered mutations at the four ICR-CTCF binding sites. The chromatin composition showed great polarization along the H19/Igf2 imprinted domain (Han et al., 2008; Singh et al., 2010a,b, 2011). Whereas the H19 gene, promoter, and ICR were enriched in active chromatin marks, H3K4me2, $\mathrm{H} 3 \mathrm{~K} 4 \mathrm{me} 3$, and $\mathrm{H} 3 \mathrm{~K} 9 \mathrm{ac}$ in the maternal allele, the paternal allele of the same regions was enriched in repressive chromatin marks, such as $\mathrm{H} 3 \mathrm{~K} 9 \mathrm{me} 3$ and $\mathrm{H} 3 \mathrm{~K} 79 \mathrm{me} 3$. The ICR was slightly maternally biased for H3K4ac, H3K18ac, H3K36ac, H3K79ac, H4K5ac, H4K8ac, H4K12ac, and H4K91ac marks, but showed biallelic H3K27me3 enrichment. The Igf2 promoter, DMR1 and DMR2 regions, were enriched in active marks, H3K4me2, H3K4me3, H3K9ac, H3K4ac, H3K18ac, H3K36аc, H3K79ac, H4K5ac, H4K8ac, H4K12ac, H4K91ac, H3K79me1, and H3K79me2 in the paternal allele but repressive marks, H3K27me3, H3K9me3 and repressive histone variant macroH2A1 in the maternal allele. 


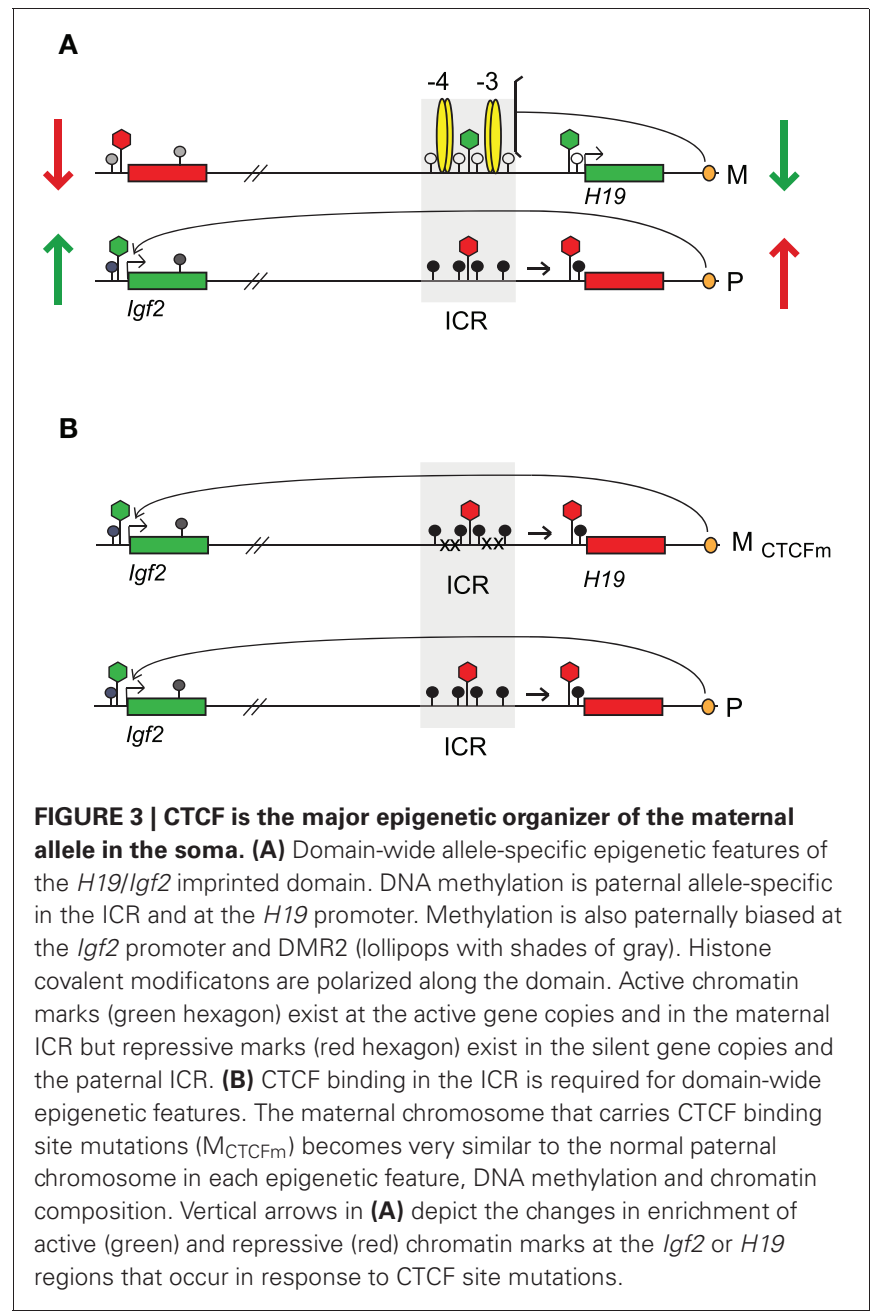

Abolishing CTCF binding in the H19-Igf2 ICR in the mutant cells resulted in a complete reorganization of the allele-specific chromatin composition (Han et al., 2008). In the maternal allele CTCF site mutant cells exhibited reduced H3K9ac, H3K4me2, and $\mathrm{H} 3 \mathrm{~K} 4 \mathrm{me} 3$ at the $\mathrm{H} 19 \mathrm{ICR}$, promoter, gene body and reduced H3K27me3 at the Igf2 P2 promoter and Igf2 DMRs. These results revealed that ICR-CTCF binding is required for recruiting the maternal allele-specific active marks, $\mathrm{H} 3 \mathrm{~K} 9 \mathrm{ac}, \mathrm{H} 3 \mathrm{~K} 4 \mathrm{me} 2$, and $\mathrm{H} 3 \mathrm{~K} 4 \mathrm{me} 3$ at the $\mathrm{H} 19$ locus and the maternal allele-specific repressing mark $\mathrm{H} 3 \mathrm{~K} 27 \mathrm{me} 3$ and macroH2A 1 at the Igf 2 locus. In agreement with these findings, it was shown that active histone tail modifications at the $H 19$ promoter depend on the activity state of the promoter (Verona et al., 2008) and that CTCF directly recruits the polycomb protein Suz12 to the Igf2 locus to catalyze H3K27 trimethylation ( $\mathrm{Li}$ et al., 2008a). In the paternal allele $\mathrm{H} 3 \mathrm{~K} 27 \mathrm{me} 3$ and macroH2A1 levels increased and became biallelic in the CTCF site-mutant cells at the H19 promoter while paternal $\mathrm{H} 3 \mathrm{~K} 4 \mathrm{me} 2$ and $\mathrm{H} 3 \mathrm{~K} 9 \mathrm{ac}$ increased and became biallelic at the Igf2 DMRs. Indeed, histone acetylation at each lysine residue increased and became biallelic in the mutant cells at the Igf2 DMR1, P2 promoter and DMR2, where it was paternal allele-specific in normal cells (Singh et al., 2010a). These results provided evidence that in the absence of CTCF binding, the mutant maternal chromosome accumulates histone marks that normally exist in the paternal chromosome. Therefore, CTCF binding in the ICR is required for excluding repressive chromatin from the $H 19$ region and excluding active chromatin, such as histone acetylation from the maternal allele at the $I g f 2$ locus at a distance.

When we examined how CTCF binding affects the histone globular domain modifications in the H19-Igf2 imprinted domain (Singh et al., 2010b), we found that the ICR CTCF site point mutations caused a twofold increase in the heterochromatin mark $\mathrm{H} 3 \mathrm{~K} 79 \mathrm{me} 3$ at the ICR sequences. Whereas it was strongly paternal allele-specific in normal cells, H3K79me3 became biallelic in the mutant cells at the ICR and at the H19 promoter, providing evidence that at these sequences CTCF is required for excluding $\mathrm{H} 3 \mathrm{~K} 79 \mathrm{me} 3$ from the maternal allele. The ICR CTCF site point mutations also caused a twofold increase of H3K79me1 and H3K79me2 levels in the mutant cells at the Igf2 P2 promoter and Igf2 DMRs where these paternal allelespecific activating chromatin marks became biallelic. H3K79me1 and $\mathrm{H} 3 \mathrm{~K} 79 \mathrm{me} 2$ levels were low in abundance and biallelic at the H19 locus and H3K79me3 levels were relatively high and biallelic at the Igf2 regions, but these features did not change in response to the CTCF site mutations, indicating that CTCF-ICR binding is not responsible in the maternal allele for including $\mathrm{H} 3 \mathrm{~K} 79 \mathrm{me} 2$ at the $\mathrm{H} 19$ region and H3K79me3 at the Igf2 locus. Taken together, with regard to globular domain modifications, the ICR CTCF site mutations have caused the paternalization of the maternal allele's chromatin composition along the H19/Igf2 imprinted domain by exclusion: CTCF was responsible for the maternal allele's chromatin composition by excluding H4K91ac, H3K79me1, and H3K79me2 at the Igf2 locus and by excluding $\mathrm{H} 3 \mathrm{~K} 79 \mathrm{me} 3$ at the $\mathrm{H} 19$ locus from the maternal allele.

In summary, with regard to histone tail modifications, in the maternal allele CTCF binding recruited active chromatin at the H19 locus and repressive chromatin at the Igf2 locus, and also excluded repressive chromatin at the $\mathrm{H} 19$ locus and active chromatin from the Igf2 locus (Han et al., 2008; Singh et al., 2010a). However, CTCF did not recruit globular domain modifications to the maternal allele, rather excluded them from the maternal allele at the Igf2 locus (Singh et al., 2010b). It will be important to find out the mechanism of how CTCF interacts with different epigenetic modifiers in achieving the maternal allele's epiphenotype.

\section{CONTROL OF DNA METHYLATION AT THE DMR}

The key to all other parental allele-specific features at the H19Igf 2 imprinted domain is the paternal-specific methylation of the ICR, because this determines monoallelic CTCF binding, and in turn CTCF binding determines monoallelic gene expression and maintenance of the polarized epigenetic features. It is important, therefore, to review here the imprint cycle of the ICR and discuss how this cycle is related to CTCF. The methylation mark in the H19/Igf2 ICR is erased between generations in PGCs (Hajkova et al., 2002) and is subsequently reestablished specifically in male fetal germ cells (Davis et al., 1999, 2000; Ueda et al., 2000; Kato et al., 2007). After that ICR methylation is maintained throughout 
spermatogenesis, fertilization, global epigenomic reprogramming in the zygote, preimplantation, and later during cell divisions in the soma (Li et al., 1993; Tucker et al., 1996; Hirasawa et al., 2008).

It is not known what initiates the paternal-specific methylation at the H19-Igf2 DMR in the male germ line, but it depends on the de novo methyltransferase Dnmt3a and its cofactor, Dnmt3L (Bourc'his et al., 2001; Kato et al., 2007; Kaneda, 2011). Even though the CTCF binding sites maintain allele-specific methylation differences in the soma (see below), the same sites are not required for setting the gametic imprint in the germ line. The ICR that harbors CTCF site mutations is fully methylated in perinatal male fetal germ cells and is fully unmethylated in fetal female germ cells and ovulated oocytes (Schoenherr et al., 2003; Szabó et al., 2004). CTCF protein may affect the maintenance of unmethylated ICR in the oocyte indirectly, because CTCFdepleted oocytes exhibit increased methylation at that region (Fedoriw et al., 2004). The methylation imprinting process at the ICR in the male germ line appears to depend on two components, the ICR sequences and also the location of the ICR inside the H19-Igf2 domain. The $(\mathrm{Ch} \beta \mathrm{GI})_{2}$ and the $(\mathrm{mCh} \beta \mathrm{GI})_{2}$ inserts (Figures 1C and D) attained only 11 and 32\% methylation in place of the ICR in 18.5 days post-coitum (dpc) prospermatogonia, respectively, suggesting that ICR sequences are important for full methylation establishment in the male germ line (Szabó et al., 2002; Lee et al., 2010). When the ICR was introduced to other genomic locations, methylation imprint establishment did not occur in the male germ line, but paternal allele-specific methylation was acquired only later in the soma. However, when the ICR was placed downstream of the $\mathrm{H} 19$ gene, it attained de novo methylation in the male germ line (Park et al., 2004; Tanimoto et al., 2005; Matsuzaki et al., 2009, 2010; Gebert et al., 2010). These studies suggested that the H19-Igf2 domain's genomic location is also important for proper imprint establishment of the H19-Igf2 ICR. It will be important to find the DNA sequencesinside and outside the ICR - that are necessary and sufficient for the mechanism of methylation imprint establishment of the ICR in prospermatogonia.

After imprint establishment the methylation of the H19-Igf2 DMR is protected in the zygote's paternal pronucleus during the wave of zygotic reprogramming (Mayer et al., 2000; Gu et al., 2011; Iqbal et al., 2011; Wossidlo et al., 2011) by the PGC7 protein (Nakamura et al., 2007). PGC7 is proposed to protect the H19Igf2 DMR from $5 \mathrm{mC}$ oxidation by Tet 3 methylcytosine oxidase in a $\mathrm{H} 3 \mathrm{~K} 9 \mathrm{me} 2$-dependent manner, similarly to how PGC7 protects the female pronucleus (Nakamura et al., 2012). H3K9me2 association at this locus is inherited from the sperm and may be sufficient to attract tight PGC7 binding, which in turn is expected to reduce Tet3 affinity to these regions (Nakamura et al., 2012). The repressor protein MBD3 is slightly biased toward the paternal allele of the ICR in ES cells and, according to MBD3 knockdown experiments, contributes to protecting CpG methylation of the paternal allele of the H19-Igf2 DMR during preimplantation development (Reese et al., 2007). Genetic studies revealed that two additional proteins protect the ICR methylation during early development. Zfp57 transcription factor protects the ICR in ES cells (Zuo et al., 2012) and Trim28 (also known as KAP1) protects it in the embryo (Messerschmidt et al., 2012). Trim28 binds to the ICR in midgestation stage embryos (Messerschmidt et al., 2012). Both Zfp57 and Trim 28 are associated with the methylated paternal allele of the ICR In ES cells (Quenneville et al., 2011). Zfp57-Trim28-Setdb1 triple occupied ChIP-sequencing peaks defined a consensus hexanucleotide sequence, TGC ${ }^{\mathrm{m}} \mathrm{CGC}$ where the CpG site is methylated (Quenneville et al., 2011). This consensus is present at each DMR, including the H19-Igf2 ICR.

In somatic organs, the maternal allele's epigenetic profile at the H19-Igf2 domain depends on CTCF binding in the ICR. CTCF binding is responsible for protecting the maternal allele from DNA methylation (Figure 3). Maternal inheritance of mutations in the CTCF binding sites resulted in highly elevated $\mathrm{CpG}$ methylation levels in somatic organs at the ICR (Pant et al., 2003; Schoenherr et al., 2003; Szabó et al., 2004), as well as the H19 promoter, and H19 gene body and even at the Igf2 DMR1 and DMR2 sequences at $\sim 90-\mathrm{kb}$ distance (Kurukuti et al., 2006; Han et al., 2008).

It is interesting to note that the Zfp57-Trim28-Setdb1 consensus sites overlap with three CTCF binding motifs in the ICR (Figure 4). At these sites the maternal allele has robust in vivo CTCF footprints in MEF. However, in MEFs no clear DNAseI footprints are discernable in the paternal allele (Szabó et al., 2000). Zfp57-Trim 28 binding may only take place in the ICR at earlier time points, before the time of MEF derivation. Incidentally, the Zfp57-Trim28-Setdb1 consensus sites have been mutated in the H19-Igf2 ICR (well before the consensus site was discovered) at the endogenous locus and in integrated transgenes (Engel et al., 2004; Matsuzaki et al., 2010). These mutations destroyed the Zfp57-Trim28-Setdb1 consensus sites such way that CTCF binding was not affected (Figure 4). As a result, methylation was reduced and insulator activity was gained in the mutant paternal ICR, likely because the reduced DNA methylation allowed CTCF binding. Zfp57-Trim28 may protect the ICR from demethylation by attracting repressing

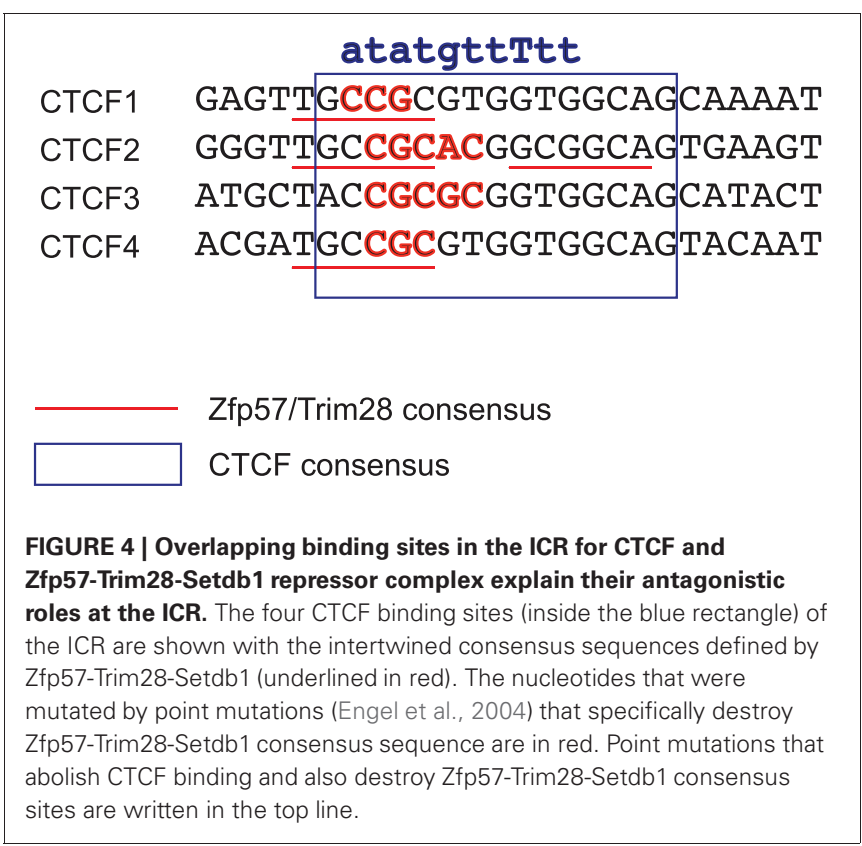


epigenetic modifiers and DNMTs to the target sequences and by facilitating heterochromatinization and DNA remethylation (Quenneville et al., 2011; Zuo et al., 2012), although this function may be redundant, because the Zfp57 null mutant midgestation embryos did not exhibit reduced ICR DNA methylation (Li et al., 2008b). It is interesting that Zfp57-Trim28-mediated protection of DNA methylation is required in the H19-Igf2 ICR only when CTCF binding sites are present. When the CTCF consensus was destroyed together with the Zfp57-Trim28-Setdb1 consensus (Figure 4), DNA methylation maintenance was not affected (Szabó et al., 2004). Zfp57-Trim28's role at the ICR, therefore, is specific to preventing CTCF binding in the paternal allele by maintaining DNA methylation. One extension of this idea is that CTCF may protect the maternal allele from DNA methylation by preventing Zfp57-Trim28-Setdb1 binding. Therefore, the antagonistic roles (Engel et al., 2004) of the composite ICR CTCF sites are the following: to maintain the methylation-free status of the maternal chromosome through CTCF binding and to maintain DNA methylation in the paternal chromosome through Zfp57-Trim28-Setdb1 binding.

\section{CTCF-DEPENDENT CHROMATIN BIAS DELAYS de novo METHYLATION OF THE MATERNAL ICR ALLELE IN MALE GERM CELLS}

The process of methylation imprint erasure at the ICR is complete in PGCs by $13.5 \mathrm{dpc}$ (Figure 5). Consequently, male fetal germ cells undergo de novo methylation at the ICR during fetal development, whereas female germ cells remain unmethylated till the end of oocyte maturation. It was noticed by several laboratories that the two ICR alleles are different in male germ cells with respect to the speed of de novo methylation. Methylation of the paternally inherited ICR allele precedes the maternally inherited allele (Davis et al., 1999, 2000; Ueda et al., 2000; Kato et al., 2007), implying that the two alleles are distinguished by an epigenetic mark, other than DNA methylation in $13.5 \mathrm{dpc}$ prospermatogonia. We hypothesized that the chromatin composition may constitute this transient epigenetic memory and this in turn depends on maternal-allele-specific binding of CTCF in PGCs. In order to test our hypothesis we isolated fetal germ cells from mice that carry SNPs at the ICR to distinguish the parental chromosomes. Using allele-specific ChIP-SNuPE and real-time reverse-transcription PCR assays we found that CTCF was slightly biased toward the maternal allele, but it had a very low level of enrichment at $13.5 \mathrm{dpc}$ at the ICR, suggesting that CTCF is almost completely removed from the ICR in germ cells before midgestation. The repressive histone mark, $\mathrm{H} 3 \mathrm{~K} 9 \mathrm{me} 3$, was slightly biased toward the paternal allele at the ICR but its enrichment level was very low whereas the active mark, $\mathrm{H} 3 \mathrm{~K} 4 \mathrm{me} 2$ was more abundant and it was slightly biased toward the maternal allele in prospermatogonia at 13.5 and $15.5 \mathrm{dpc}$. The level of $\mathrm{H} 3 \mathrm{~K} 4 \mathrm{me} 2$ allelic bias was similar to the methylation bias between alleles (10$15 \%)$. When the maternal allele carried the CTCF site mutations in prospermatogonia, the chromatin bias was no longer observed at the ICR, suggesting that chromatin composition of the ICR depends on maternal-allele specific CTCF binding in PGCs, just like it does in somatic cells (Han et al.,

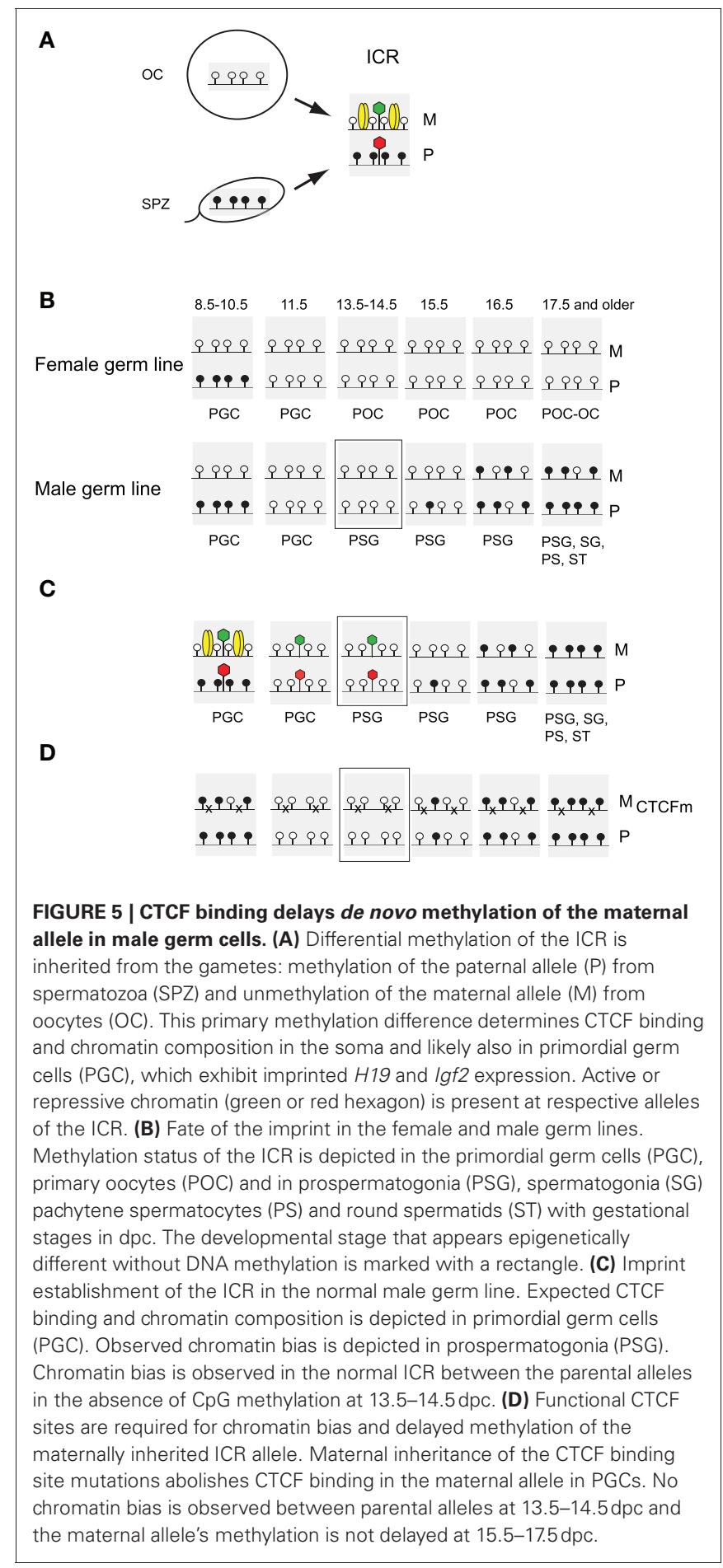

2008; Singh et al., 2010a,b). The methylation bias was also absent between the parental alleles in the mutant prospermatogonia. These findings are consistent with the explanation that CTCF binding in PGCs is responsible for setting up a chromatin bias in PGCs, and that this chromatin is not fully erased in prospermatogonia before de novo methylation commences. Therefore, CTCF-dependent chromatin bias may influence the rate of DNA methylation in the parental alleles. We concluded 
that it is the H3K4me2 histone mark that most likely constitutes the epigenetic memory of the mother in prospermatogonia at 13.5-14.5 dpc and delays de novo CpG methylation in the maternal ICR allele. Indeed, removal of $\mathrm{H} 3 \mathrm{~K} 4 \mathrm{me} 2$ by $\mathrm{H} 3 \mathrm{~K} 4$ demethylase KDM1B is required at least at certain maternal DMRs for the establishment of methylation imprints in oocytes (Ciccone et al., 2009). It is known that certain maternal DMRs exhibit delayed de novo methylation in the paternally inherited allele (Hiura et al., 2006). It will be interesting to find out using genetic analyses whether CTCF or other transcription factor provides transient epigenetic memory for those alleles.

In summary, CTCF plays complex roles at the H19-Igf2 ICR. All of these roles may appear at first to depend on its major role at the domain, which is enhancer blocking. However, CTCF also protects the ICR from DNA methylation in the maternal allele and also sets up the maternal allele's chromatin composition in the soma and to some extent in PGCs. These functions at a single locus illuminate the versatility of CTCF in organizing gene expression and also in structuring the genome. It will be important to carry out similar genetic experiment by precisely inactivating the binding sites using point mutations to understand whether CTCF organizes local and domain-wide chromatin composition and/or maintains the unmethylated state at other loci in the genome, especially those that where insulator function has been shown (Herold et al., 2012). At least at one other

\section{REFERENCES}

Abramowitz, L. K., and Bartolomei, M. S. (2012). Genomic imprinting: recognition and marking of imprinted loci. Curr. Opin. Genet. Dev. 22, 72-78.

Barkess, G., and West, A. G. (2012). Chromatin insulator elements: establishing barriers to set heterochromatin boundaries. Epigenomics 4, 67-80.

Bell, A. C., and Felsenfeld, G. (2000). Methylation of a CTCF-dependent boundary controls imprinted expression of the Igf 2 gene. Nature 405, 482-485.

Bergstrom, R., Whitehead, J., Kurukuti, S., and Ohlsson, R. (2007). CTCF regulates asynchronous replication of the imprinted H19/Igf2 domain. Cell Cycle 6, 450-454.

Bourc'his, D., Xu, G. L., Lin, C. S., Bollman, B., and Bestor, T. H. (2001). Dnmt3L and the establishment of maternal genomic imprints. Science 294, 2536-2539.

Ciccone, D. N., Su, H., Hevi, S., Gay, F., Lei, H., Bajko, J., et al. (2009). KDM1B is a histone H3K4 demethylase required to establish maternal genomic imprints. Nature 461, 415-418.

Clark, S. P., Lewis, C. D., and Felsenfeld, G. (1990). Properties of BGP1, a poly $(\mathrm{dG})$-binding protein from chicken erythrocytes. Nucleic Acids Res. 18, 5119-5126.
Constancia, M., Dean, W., Lopes, S., Moore, T., Kelsey, G., and Reik, W. (2000). Deletion of a silencer element in Igf2 results in loss of imprinting independent of $\mathrm{H} 19$. Nat. Genet. 26, 203-206.

Constancia, M., Hemberger, M., Hughes, J., Dean, W., FergusonSmith, A., Fundele, R., et al. (2002). Placental-specific IGF-II is a major modulator of placental and fetal growth. Nature 417, 945-948.

Davis, T. L., Trasler, J. M., Moss, S. B., Yang, G. J., and Bartolomei, M. S. (1999). Acquisition of the H19 methylation imprint occurs differentially on the parental alleles during spermatogenesis. Genomics 58, $18-28$.

Davis, T. L., Yang, G. J., McCarrey, J. R., and Bartolomei, M. S. (2000). The H19 methylation imprint is erased and re-established differentially on the parental alleles during male germ cell development. Hum. Mol. Genet. 9, 2885-2894.

DeChiara, T. M., Efstratiadis, A., and Robertson, E. J. (1990). A growthdeficiency phenotype in heterozygous mice carrying an insulin-like growth factor II gene disrupted by targeting. Nature 345, 78-80.

Dickson, J., Gowher, H., Strogantsev, R., Gaszner, M., Hair, A., Felsenfeld, G., et al. (2010). VEZF1 elements mediate

locus, at the $\beta$-globin cluster $3^{\prime} \mathrm{HS} 1$, CTCF binding was shown to be required for recruiting active chromatin mark $\mathrm{H} 3 \mathrm{~K} 9 \mathrm{ac}$ and repelling the repressing marks H3K9/27me3 (Splinter et al., 2006). We will be very curious to see whether CTCF binding sites in the Xist/Tsix RS14 region (Spencer et al., 2011) regulate the choice of X chromosome for inactivation by orchestrating local or domain-wide chromatin composition. Interestingly, mutations in the corresponding human sites either increase or decrease CTCF binding affinity and also reciprocally affect $\mathrm{X}$ inactivation skewing (Pugacheva et al., 2005). It will be especially critical to find out whether CTCF carries out its chromatin organizing activities parental allele-specifically at other imprinted domains and if proper CTCF binding at those DMRs is essential for development. We expect that this will be true at least at the Dlk1-Gtl2 imprinted domain, because CTCF binding is allele-specific in a strategically important location at the Gtl2 promoter (Lin et al., 2011) and because of the known lethality phenotypes associated with the misregulation of allele-specific expression at this imprinted domain (Lin et al., 2003; Wu et al., 2006; Takahashi et al., 2009, 2010).

\section{ACKNOWLEDGMENTS}

This work was supported by a Public Health Service grant (GM064378) from the National Institute of General Medicine to Piroska E. Szabó.

protection from DNA methylation. PLoS Genet. 6:e1000804. doi: 10.1371/journal.pgen.1000804

Engel, N., West, A. G., Felsenfeld, G., and Bartolomei, M. S. (2004). Antagonism between DNA hypermethylation and enhancer-blocking activity at the H19 DMD is uncovered by CpG mutations. Nat. Genet. 36, 883-888.

Fedoriw, A. M., Stein, P., Svoboda, P., Schultz, R. M., and Bartolomei, M. S. (2004). Transgenic RNAi reveals essential function for CTCF in $\mathrm{H} 19$ gene imprinting. Science 303 , 238-240.

Ferguson-Smith, A. C. (2011) Genomic imprinting: the emergence of an epigenetic paradigm. Nat. Rev. Genet. 12, 565-575.

Filippova, G. N. (2008). Genetics and epigenetics of the multifunctional protein CTCF. Curr. Top. Dev. Biol. 80, 337-360.

Fitzpatrick, G. V., Pugacheva, E. M., Shin, J. Y., Abdullaev, Z., Yang, Y., Khatod, K., et al. (2007). Allelespecific binding of CTCF to the multipartite imprinting control region KvDMR1. Mol. Cell. Biol. 27, 2636-2647.

Gabory, A., Ripoche, M. A., Le Digarcher, A., Watrin, F., Ziyyat, A., Forne, T., et al. (2009). H19 acts as a trans regulator of the imprinted gene network controlling growth in mice. Development 136, 3413-3421.
Gebert, C., Kunkel, D., Grinberg, A., and Pfeifer, K. (2010). H19 imprinting control region methylation requires an imprinted environment only in the male germ line. Mol. Cell. Biol. 30, 1108-1115.

Ghirlando, R., Giles, K., Gowher, H., Xiao, T., Xu, Z., Yao, H., et al. (2012). Chromatin domains, insulators, and the regulation of gene expression. Biochim. Biophys. Acta 1819, 644-651.

Gu, T. P., Guo, F., Yang, H., Wu, H P., Xu, G. F., Liu, W., et al. (2011). The role of Tet3 DNA dioxygenase in epigenetic reprogramming by oocytes. Nature 477, 606-610.

Guibert, S., Zhao, Z., Sjolinder, M., Gondor, A., Fernandez, A., Pant, V., et al. (2012). CTCF-binding sites within the H19 ICR differentially regulate local chromatin structures and cis-acting functions. Epigenetics 7, 361-369.

Guo, C., Yoon, H. S., Franklin, A., Jain, S., Ebert, A., Cheng, H. L., et al. (2011). CTCF-binding elements mediate control of V(D)J recombination. Nature 477, 424-430.

Hajkova, P., Erhardt, S., Lane, N., Haaf, T., El-Maarri, O., Reik, W., et al. (2002). Epigenetic reprogramming in mouse primordial germ cells. Mech. Dev. 117, 15-23.

Han, L., Lee, D. H., and Szabó, P. E. (2008). CTCF is the master 
organizer of domain-wide allelespecific chromatin at the H19/Igf2 imprinted region. Mol. Cell. Biol. 28, 1124-1135.

Han, L., Szabó, P. E., and Mann, J. R. (2010). Postnatal survival of mice with maternal duplication of distal chromosome 7 induced by a Igf2/H19 imprinting control region lacking insulator function. PLoS Genet. 6:e1000803. doi: 10.1371/journal.pgen.1000803

Hark, A. T., Schoenherr, C. J., Katz, D. J., Ingram, R. S., Levorse, J. M., and Tilghman, S. M. (2000). CTCF mediates methylation-sensitive enhancer-blocking activity at the H19/Igf2 locus. Nature 405, 486-489.

Herold, M., Bartkuhn, M., and Renkawitz, R. (2012). CTCF: insights into insulator function during development. Development 139, 1045-1057.

Hikichi, T., Kohda, T., KanekoIshino, T., and Ishino, F. (2003). Imprinting regulation of the murine Meg1/Grb10 and human GRB10 genes; roles of brain-specific promoters and mouse-specific CTCF-binding sites. Nucleic Acids Res. 31, 1398-1406.

Hirasawa, R., Chiba, H., Kaneda, M., Tajima, S., Li, E., Jaenisch, R., et al. (2008). Maternal and zygotic Dnmtl are necessary and sufficient for the maintenance of DNA methylation imprints during preimplantation development. Genes Dev. 22, 1607-1616.

Hiura, H., Obata, Y., Komiyama, J., Shirai, M., and Kono, T. (2006). Oocyte growth-dependent progression of maternal imprinting in mice. Genes Cells 11, 353-361.

Iqbal, K., Jin, S. G., Pfeifer, G. P., and Szabó, P. E. (2011). Reprogramming of the paternal genome upon fertilization involves genome-wide oxidation of 5-methylcytosine. Proc. Natl. Acad. Sci. U.S.A. 108, 3642-3647.

Kanduri, C., Pant, V., Loukinov, D., Pugacheva, E., Qi, C. F., Wolffe, A., et al. (2000). Functional association of CTCF with the insulator upstream of the $\mathrm{H} 19$ gene is parent of origin-specific and methylationsensitive. Curr. Biol. 10, 853-856.

Kaneda, M. (2011). Genomic imprinting in mammals-epigenetic parental memories. Differentiation 82, $51-56$.

Kato, Y., Kaneda, M., Hata, K., Kumaki, K., Hisano, M., Kohara, Y., et al. (2007). Role of the Dnmt3 family in de novo methylation of imprinted and repetitive sequences during male germ cell development in the mouse. Hum. Mol. Genet. 16, 2272-2280.

Keniry, A., Oxley, D., Monnier, P., Kyba, M., Dandolo, L., Smits, G., et al. (2012). The H19 lincRNA is a developmental reservoir of miR-675 that suppresses growth and Igflr. Nat. Cell Biol. 14, 659-665.

Kurukuti, S., Tiwari, V. K., Tavoosidana, G., Pugacheva, E., Murrell, A., Zhao, Z., et al. (2006). CTCF binding at the H19 imprinting control region mediates maternally inherited higher-order chromatin conformation to restrict enhancer access to Igf2. Proc. Natl. Acad. Sci. U.S.A. 103, 10684-10689.

Lee, D. H., Singh, P., Tsark, W. M., and Szabó, P. E. (2010). Complete biallelic insulation at the H19/Igf2 imprinting control region position results in fetal growth retardation and perinatal lethality. PLoS ONE 5:e12630. doi: 10.1371/journal.pone. 0012630

Leighton, P. A., Saam, J. R., Ingram, R. S., Stewart, C. L., and Tilghman, S. M. (1995). An enhancer deletion affects both $\mathrm{H} 19$ and Igf2 expression. Genes Dev. 9, 2079-2089.

Li, E., Beard, C., and Jaenisch, R. (1993). Role for DNA methylation in genomic imprinting. Nature 366, 362-365.

Li, T., Hu, J. F., Qiu, X., Ling, J., Chen, H., Wang, S., et al. (2008a). CTCF regulates allelic expression of Igf2 by orchestrating a promoter-polycomb repressive complex 2 intrachromosomal loop. Mol. Cell. Biol. 28, 6473-6482.

Li, X., Ito, M., Zhou, F., Youngson, N., Zuo, X., Leder, P., et al. (2008b). A maternal-zygotic effect gene, Zfp57, maintains both maternal and paternal imprints. Dev. Cell 15, 547-557.

Libby, R. T., Hagerman, K. A., Pineda, V. V., Lau, R., Cho, D. H., Baccam, S. L., et al. (2008). CTCF cis-regulates trinucleotide repeat instability in an epigenetic manner: a novel basis for mutational hot spot determination. PLoS Genet. 4:e1000257. doi: 10.1371/journal.pgen. 1000257

Lin, S., Ferguson-Smith, A. C., Schultz, R. M., and Bartolomei, M. S. (2011). Nonallelic transcriptional roles of CTCF and cohesins at imprinted loci. Mol. Cell. Biol. 31, 3094-3104.

Lin, S. P., Youngson, N., Takada, S., Seitz, H., Reik, W., Paulsen, M., et al. (2003). Asymmetric regulation of imprinting on the maternal and paternal chromosomes at the Dlk1-Gtl2 imprinted cluster on mouse chromosome 12. Nat. Genet. 35, 97-102.
Matsuzaki, H., Okamura, E., Fukamizu, A., and Tanimoto, K. (2010). CTCF binding is not the epigenetic mark that establishes post-fertilization methylation imprinting in the transgenic H19 ICR. Hum. Mol. Genet. 19, 1190-1198.

Matsuzaki, H., Okamura, E. Shimotsuma, M., Fukamizu, A., and Tanimoto, K. (2009). A randomly integrated transgenic H19 imprinting control region acquires methylation imprinting independently of its establishment in germ cells. Mol. Cell. Biol. 29, 4595-4603.

Mayer, W., Niveleau, A., Walter, J., Fundele, R., and Haaf, T. (2000) Demethylation of the zygotic paternal genome. Nature 403, 501-502.

Messerschmidt, D. M., de Vries, W. Ito, M., Solter, D., Ferguson-Smith, A., and Knowles, B. B. (2012). Trim28 is required for epigenetic stability during mouse oocyte to embryo transition. Science 335, 1499-1502.

Moore, J. M., Rabaia, N. A., Smith, L. E., Fagerlie, S., Gurley, K., Loukinov, D., et al. (2012). Loss of maternal CTCF is associated with periimplantation lethality of Ctcf null embryos. PLoS ONE 7:e34915. doi: 10.1371/journal.pone.0034915

Murrell, A. (2011). Setting up and maintaining differential insulators and boundaries for genomic imprinting. Biochem. Cell Biol. 89, 469-478.

Murrell, A., Heeson, S., Bowden, L., Constancia, M., Dean, W., Kelsey, G., et al. (2001). An intragenic methylated region in the imprinted Igf2 gene augments transcription. EMBO Rep. 2, 1101-1106.

Nakamura, T., Arai, Y., Umehara, H., Masuhara, M., Kimura, T., Taniguchi, H., et al. (2007). PGC7/Stella protects against DNA demethylation in early embryogenesis. Nat. Cell Biol. 9, 64-71.

Nakamura, T., Liu, Y.-J., Nakashima, H., Umehara, H., Inoue, K., Matoba, S., et al. (2012). PGC7 binds histone $\mathrm{H} 3 \mathrm{~K} 9 \mathrm{me} 2$ to protect against conversion of $5 \mathrm{mC}$ to $5 \mathrm{hmC}$ in early embryos. Nature 486, 415-419.

Nativio, R., Wendt, K. S., Ito, Y., Huddleston, J. E., Uribe-Lewis, S., Woodfine, K., et al. (2009). Cohesin is required for higherorder chromatin conformation at the imprinted IGF2-H19 locus. PLoS Genet. 5:e1000739. doi: 10.1371/journal.pgen.1000739

Ohlsson, R., Bartkuhn, M., and Renkawitz, R. (2010). CTCF shapes chromatin by multiple mechanisms: the impact of 20 years of CTCF research on understanding the workings of chromatin. Chromosoma 119, 351-360.

Pant, V., Mariano, P., Kanduri, C., Mattsson, A., Lobanenkov, V., Heuchel, R., et al. (2003). The nucleotides responsible for the direct physical contact between the chromatin insulator protein CTCF and the H19 imprinting control region manifest parent of origin-specific long-distance insulation and methylation-free domains. Genes Dev. 17, 586-590.

Park, K. Y., Sellars, E. A., Grinberg, A., Huang, S. P., and Pfeifer, K. (2004). The H19 differentially methylated region marks the parental origin of a heterologous locus without gametic DNA methylation. Mol. Cell. Biol. 24, 3588-3595.

Phillips, J. E., and Corces, V. G. (2009). CTCF: master weaver of the genome. Cell 137, 1194-1211.

Pugacheva, E. M., Tiwari, V. K., Abdullaev, Z., Vostrov, A. A., Flanagan, P. T., Quitschke, W. W., et al. (2005). Familial cases of point mutations in the XIST promoter reveal a correlation between CTCF binding and pre-emptive choices of $\mathrm{X}$ chromosome inactivation. Hum. Mol. Genet. 14, 953-965.

Quenneville, S., Verde, G., Corsinotti, A., Kapopoulou, A., Jakobsson, J., Offner, S., et al. (2011). In embryonic stem cells, ZFP57/KAP1 recognize a methylated hexanucleotide to affect chromatin and DNA methylation of imprinting control regions. Mol. Cell 44, 361-372.

Reese, K. J., Lin, S., Verona, R. I. Schultz, R. M., and Bartolomei, M. S. (2007). Maintenance of paternal methylation and repression of the imprinted $\mathrm{H} 19$ gene requires MBD3. PLoS Genet. 3:e137. doi: 10.1371/journal.pgen.0030137

Rubio, E. D., Reiss, D. J., Welcsh, P. L., Disteche, C. M., Filippova, G. N., Baliga, N. S., et al. (2008). CTCF physically links cohesin to chromatin. Proc. Natl. Acad. Sci. U.S.A. 105, 8309-8314.

Schoenherr, C. J., Levorse, J. M., and Tilghman, S. M. (2003). CTCF maintains differential methylation at the Igf2/H19 locus. Nat. Genet. 33, 66-69.

Shukla, S., Kavak, E., Gregory, M., Imashimizu, M., Shutinoski, B. Kashlev, M., et al. (2011). CTCFpromoted RNA polymerase II pausing links DNA methylation to splicing. Nature 479, 74-79.

Singh, P., Cho, J., Tsai, S. Y., Rivas, G. E., Larson, G. P., and Szabó 
P. E. (2010a). Coordinated allelespecific histone acetylation at the differentially methylated regions of imprinted genes. Nucleic Acids Res. 38, 7974-7990.

Singh, P., Han, L., Rivas, G. E., Lee, D. H., Nicholson, T. B., Larson, G. P., et al. (2010b). Allele-specific H3K79 Di- versus trimethylation distinguishes opposite parental alleles at imprinted regions. Mol. Cell. Biol. 30, 2693-2707.

Singh, P., Wu, X., Lee, D. H., Li, A. X., Rauch, T. A., Pfeifer, G. P., et al. (2011). Chromosome-wide analysis of parental allele-specific chromatin and DNA methylation. Mol. Cell. Biol. 31, 1757-1770.

Spencer, R. J., del Rosario, B. C., Pinter, S. F., Lessing, D., Sadreyev, R. I., and Lee, J. T. (2011). A boundary element between Tsix and Xist binds the chromatin insulator Ctcf and contributes to initiation of $\mathrm{X}$ chromosome inactivation. Genetics 189, 441-454.

Splinter, E., Heath, H., Kooren, J., Palstra, R. J., Klous, P., Grosveld, F., et al. (2006). CTCF mediates long-range chromatin looping and local histone modification in the beta-globin locus. Genes Dev. 20, 2349-2354.

Stedman, W., Kang, H., Lin, S., Kissil, J. L., Bartolomei, M. S., and Lieberman, P. M. (2008). Cohesins localize with CTCF at the KSHV latency control region and at cellular $\mathrm{c}-\mathrm{myc}$ and H19/Igf2 insulators. EMBO J. 27, 654-666.

Szabó, P., Tang, S. H., Rentsendorj, A., Pfeifer, G. P., and Mann, J. R. (2000). Maternal-specific footprints at putative CTCF sites in the H19 imprinting control region give evidence for insulator function. Curr. Biol. 10, 607-610.

Szabó, P. E., Tang, S. H., Reed, M. R., Silva, F. J., Tsark, W. M., and Mann, J. R. (2002). The chicken beta-globin insulator element conveys chromatin boundary activity but not imprinting at the mouse
Igf2/H19 domain. Development 129, 897-904.

Szabó, P. E., Tang, S. H., Silva, F. J., Tsark, W. M., and Mann, J. R. (2004). Role of CTCF binding sites in the Igf2/H19 imprinting control region. Mol. Cell. Biol. 24, 4791-4800.

Takahashi, N., Kobayashi, R., and Kono, T. (2010). Restoration of Dlk1 and Rtll is necessary but insufficient to rescue lethality in intergenic differentially methylated region (IG-DMR)-deficient mice. J. Biol. Chem. 285, 26121-26125.

Takahashi, N., Okamoto, A., Kobayashi, R., Shirai, M., Obata, Y., Ogawa, H., et al. (2009). Deletion of Gtl2, imprinted non-coding RNA, with its differentially methylated region induces lethal parent-origindependent defects in mice. Hum. Mol. Genet. 18, 1879-1888.

Tanimoto, K., Shimotsuma, M., Matsuzaki, H., Omori, A., Bungert, J., Engel, J. D., et al. (2005). Genomic imprinting recapitulated in the human beta-globin locus. Proc. Natl. Acad. Sci. U.S.A. 102, 10250-10255.

Thorvaldsen, J. L., Duran, K. L., and Bartolomei, M. S. (1998). Deletion of the $\mathrm{H} 19$ differentially methylated domain results in loss of imprinted expression of H19 and Igf2. Genes Dev. 12, 3693-3702.

Tremblay, K. D., Duran, K. L., and Bartolomei, M. S. (1997). A $5^{\prime}$ 2-kilobase-pair region of the imprinted mouse $\mathrm{H} 19$ gene exhibits exclusive paternal methylation throughout development. Mol. Cell. Biol. 17, 4322-4329.

Tremblay, K. D., Saam, J. R., Ingram, R. S., Tilghman, S. M., and Bartolomei, M. S. (1995). A paternal-specific methylation imprint marks the alleles of the mouse H19 gene. Nat. Genet. 9, 407-413.

Tucker, K. L., Beard, C., Dausmann, J., Jackson-Grusby, L., Laird, P. W., Lei, H., et al. (1996). Germ-line passage is required for establishment of methylation and expression patterns of imprinted but not of nonimprinted genes. Genes Dev. 10, 1008-1020.

Ueda, T., Abe, K., Miura, A., Yuzuriha, M., Zubair, M., Noguchi, M., et al. (2000). The paternal methylation imprint of the mouse H19 locus is acquired in the gonocyte stage during foetal testis development. Genes Cells 5, 649-659.

Verona, R. I., Thorvaldsen, J. L., Reese, K. J., and Bartolomei, M. S. (2008). The transcriptional status but not the imprinting control region determines allele-specific histone modifications at the imprinted $\mathrm{H} 19$ locus. Mol. Cell. Biol. 28, 71-82.

West, A. G., Huang, S., Gaszner, M., Litt, M. D., and Felsenfeld, G. (2004). Recruitment of histone modifications by USF proteins at a vertebrate barrier element. Mol. Cell 16, 453-463.

Weth, O., and Renkawitz, R. (2011). CTCF function is modulated by neighboring DNA binding factors. Biochem. Cell Biol. 89, 459-468.

Wossidlo, M., Nakamura, T., Lepikhov, K., Marques, C. J., Zakhartchenko, V., Boiani, M., et al. (2011). 5-Hydroxymethylcytosine in the mammalian zygote is linked with epigenetic reprogramming. Nat. Commun. 2, 241.

Wu, Q., Kumagai, T., Kawahara, M., Ogawa, H., Hiura, H., Obata, Y., et al. (2006). Regulated expression of two sets of paternally imprinted genes is necessary for mouse parthenogenetic development to term. Reproduction 131, 481-488.

Xiao, T., Wallace, J., and Felsenfeld, G. (2011). Specific sites in the $C$ terminus of CTCF interact with the SA2 subunit of the cohesin complex and are required for cohesin-dependent insulation activity. Mol. Cell. Biol. 31, 2174-2183.

Yao, H., Brick, K., Evrard, Y., Xiao, T., Camerini-Otero, R. D., and Felsenfeld, G. (2010). Mediation of CTCF transcriptional insulation by DEAD-box RNA-binding protein p68 and steroid receptor
RNA activator SRA. Genes Dev. 24, 2543-2555.

Yoon, B., Herman, H., Hu, B., Park, Y. J., Lindroth, A., Bell, A., et al. (2005). Rasgrf1 imprinting is regulated by a CTCF-dependent methylation-sensitive enhancer blocker. Mol. Cell. Biol. 25, 11184-11190.

Yoshimizu, T., Miroglio, A., Ripoche, M. A., Gabory, A., Vernucci, M., Riccio, A., et al. (2008). The H19 locus acts in vivo as a tumor suppressor. Proc. Natl. Acad. Sci. U.S.A. 105, 12417-12422.

Zuo, X., Sheng, J., Lau, H. T., McDonald, C. M., Andrade, M., Cullen, D. E., et al. (2012). Zinc finger protein ZFP57 requires its co-factor to recruit DNA methyltransferases and maintains DNA methylation imprint in embryonic stem cells via its transcriptional repression domain. J. Biol. Chem. 287, 2107-2118.

Conflict of Interest Statement: The authors declare that the research was conducted in the absence of any commercial or financial relationships that could be construed as a potential conflict of interest.

Received: 18 July 2012; paper pending published: 13 August 2012; accepted: 27 September 2012; published online: 15 October 2012.

Citation: Singh P, Lee D-H and Szabó $P E$ (2012) More than insulator: multiple roles of CTCF at the H19-Igf2 imprinted domain. Front. Gene. 3:214. doi: 10.3389/fgene.2012.00214

This article was submitted to Frontiers in Epigenomics, a specialty of Frontiers in Genetics.

Copyright (c) 2012 Singh, Lee and Szabó. This is an open-access article distributed under the terms of the Creative Commons Attribution License, which permits use, distribution and reproduction in other forums, provided the original authors and source are credited and subject to any copyright notices concerning any third-party graphics etc. 